\title{
Screening of Salmonella enteritidis and Bacillus thuringiensis proteomes for potentially amyloidogenic proteins
}

\author{
A.O. Kosolapova ${ }^{1,2}$, K.S. Antonets ${ }^{1,2 *}$, A.A. Nizhnikov ${ }^{1,2}$ \\ ${ }^{1}$ Laboratory for Proteomics of Supra-Organismal Systems, All-Russia Research Institute for Agricultural \\ Microbiology, Pushkin, St. Petersburg, Russia \\ ${ }^{2}$ Dept. of Genetics and Biotechnology, St. Petersburg State University, St. Petersburg, Russia \\ *e-mail: kirantonez@gmail.com
}

Key words: amyloid, pathogen, bacteria

Motivation and aims: Salmonella enteritidis and Bacillus thuringiensis are pathogens in different groups of organisms. S. enteritidis causes intestinal infections of rodents and B. thuringiensis is insecticidal pathogen used to control pests. Recently it was shown that bacterial factors of virulence might form amyloids and it might play an important role in infection [1]. The aim of this study was to find potentially amyloidogenic proteins in the proteomes of $S$. enteritidis and B. thuringiensis.

Methods: To find amyloidogenic regions in proteins we used Waltz tool [2], which was developed to predict amyloid-forming regions; Aggrescan [3], which finds aggregationprone regions; and SARP algorithm [4], which was designed to find compositionally biased regions. All protein sequences and their annotations were downloaded from Uniprot database (uniprot.org).

Results: We found that about $30 \%$ of all proteins of S. enteritidis and B. thuringiensis, harbored regions predicted with Waltz, up to $1 \%$ of proteins were enriched with amyloidogenic amino acids asparagine and glutamine (QN-rich) and more than $80 \%$ of proteins contained regions found with Aggrescan. Proteins with Waltz-predicted regions are mostly membrane-associated and participate in transport or biosynthesis. The group of flagellar proteins were significantly enriched with QN-rich proteins, found with SARP. The flagellum proteins participate in host-pathogen interactions. All proteins attributed to the process of pathogenesis by GeneOntlogy database, contained regions found with Aggrescan. Conclusion: We have found that various factors of virulence of the phylogenetically distant pathogenic bacteria Salmonella enteritidis and Bacillus thuringiensis are rich in amyloidogenic regions. Thus, amyloid formation by the factors of virulence might represent evolutionary conservative molecular mechanism underlying pathogenesis of different bacterial infections.

Acknowledgements: The analysis of amyloidogenic regions with Waltz and SARP programs was supported by the Grant of the President of the Russian Federation (MK-3240.2017.4 to AAN). Prediction of aggregation-prone regions in B. thuringiensis and $S$. enteritidis within Aggrescan was supported by Budget Project (0664-2015-0018).

\section{References}

1. Belousov M.V. et al. (2018) M60-like metalloprotease domain of Escherichia coli YghJ protein forms amyloid fibrils. PLoS One. 13(1):e0191317.

2. Maurer-Stroh S. et al. (2010) Exploring the sequence determinants of amyloid structure using positionspecific scoring matrices. Nat. Methods. 7:237-242.

3. Conchillo-Sole O. et al. (2007) AGGRESCAN: a server for prediction and evaluation of "hot spots" of aggregation in polypeptides. BMC Bioinformatics. 8:65.

4. Antonets K.S., Nizhnikov A.A. (2013) SARP: a novel algorithm to assess compositional biases in protein sequences. Evol. Bioinform. 9:263-73. 